\title{
A Framework for Self-organized Network Composition
}

\author{
Cornelia Kappler ${ }^{1}$, Paulo Mendes ${ }^{2}$, Christian Prehofer $^{2}$, \\ Petteri Pöyhönen ${ }^{3}$, and Di Zhou ${ }^{4}$ \\ ${ }^{1}$ Siemens AG Communications, 13623 Berlin, Germany \\ cornelia.kappler@siemens.com \\ ${ }^{2}$ DoCoMo Euro-Labs, Munich, Germany \\ lastnamedacomolab-euro.com \\ ${ }^{3}$ Nokia Research Center, Nokia, Helsinki, Finland \\ petteri.poyhonen@nokia.com \\ ${ }^{4}$ Siemens AG Austria, A-1210 Vienna, Austria \\ di.zhou@siemens.com
}

\begin{abstract}
This paper discusses a framework for a flexible, self-organized control plane for future mobile and ubiquitous networks. The current diversification of control planes requires a manual configuration of network interworking. The problem will increase in the future, with more dynamic topologies and integration of heterogeneous networks in a ubiquitous, reactive environment. In this paper we introduce the concept of network composition, a basic, scalable and dynamic network operation to achieve autonomic control plane interworking between Ambient Networks - our approach for next generation networks. We show the architectural components of a generic control plane and its flexible interfaces. With an example on seamless mobility we illustrate how composition can simplify and improve the interworking of future networks.
\end{abstract}

Keywords: Designing evolvable NGNs, Self-organization for NGN reconfigurability.

\section{Introduction}

This paper discusses a framework for a flexible, self-organized control plane for future mobile and ubiquitous networks. When looking at the control plane of current networks, i.e. mobile cellular networks and the Internet, we have a very diverse situation. Mobile networks, based e.g. on 3GPP (3rd Generation Partnership Project) standards, have a very powerful, but also inflexible and special-purpose control plane. This means that connecting two such mobile networks, via roaming agreements, results in good interworking, but only for pre-arranged, fixed services such as voice calls, SMS (Short Message Service) or basic data services. Roaming agreements moreover need to be established manually.

On the other hand, the Internet in its current form only has a very basic control plane which enables packet routing between different networks. Hence interworking of networks is easier, but mostly provides best effort data transport. Regarding more 
advanced features, the global Internet consists of many heterogeneous networks interconnected with varying degrees of trust and cooperation: different control environments are established for services like Virtual Private Network (VPN), security, integrated mobility management, Quality of Service (QoS), Network Address Translation (NAT), and multicast. Hence, connectivity between IP networks is provided, but the control planes of those networks are often not compatible. Network interworking therefore also is typically manually configured.

In the future, more dynamic topologies and heterogeneous networks in a ubiquitous, responsive environment are expected. New kinds of mobile networks will appear, such as Personal Area Networks (PANs), Body Area Networks (BANs), intervehicle networks, and sensor networks, all of which will interwork. The control plane interaction of these networks needs to enable e.g. seamless mobility, end-to-end QoS, integrated security and accounting. For instance, mobility handling is different for a mobile phone, a train network or a BAN. Hence it needs to be negotiated which specific protocols to use and in which way. The configuration of control-plane interaction of such networks needs to become autonomic, because it is a very complex process and yet needs to be realized on-the-fly, and moreover transparently to the user. The owners of future ubiquitous networks often are non-experts and hence cannot be burdened with technical details.

Application scenarios for autonomic configuration of control-plane interaction include

- Automatically established roaming agreements between mobile operators,

- Connecting the access network of a train to access networks along the track,

- Creation of vehicular access networks with changing participants,

- Creation of a users PAN,

- Using the PAN of another user to access the Internet.

We address this problem by introducing a new framework for interworking of the next generation of networks based on work currently under way within the Integrated Project "Ambient Networks" supported by the EU [1]. In this framework, a network is viewed as a composed set of Ambient Networks (ANs) [2]. We argue that the AN concept will not only ensure the maintenance of the openness, reliability and robustness of the Internet, but will also allow an easy usage of communications services in an increasingly complex mesh of different, particularly mobile, networks. To establish control-plane interaction of networks, we introduce the concept of network composition.

We use the following two main concepts as the basis for our framework:

- End systems are seen not as nodes, but as (functionality-reduced) ANs. In the future, end-users will not just own terminal devices, but they will own and operate networks of personal devices like PANs and BANs. The notion of a network is now stretching from single devices over small, user-owned networks to globally operated networks. In this way, we can address the enormous variety of networks in a unified way. 
- Network composition is used as the basic, essential operation between AN control planes. Composition enables ANs to cooperate on the control plane; it generalizes and streamlines many existing basic concepts like attaching a node to a network, mobility of nodes and networks (viewed as changing the composition structure) as well as typical inter-operator network agreements.

The remainder of this paper is organized as follows. In Section 2, we discuss related work. In Section 3, we present possible application areas. In Section 4, we describe the concept of composition and how it could overcome today's networking limitations. Section 5 presents an example, and Section 6 draws conclusions and lines out next steps.

\section{Related Work}

The idea of control-plane interworking in a dynamic or self-organized manner has already been discussed in the literature from different perspectives. The work in [3] propagates a kind of meta-control plane, called knowledge plane, for future intelligent management of the Internet. The knowledge plane has a high-level model of what the network is supposed to do, and relies on tools of Artificial Intelligence and Cognitive Systems. In [4] a self-organizing system is proposed that supports spontaneous information exchange and service deployment in ad hoc networks based on interaction patterns between mobile ad hoc nodes. The paper also states the lack of general self-organizing mechanism for dynamic communication environments like mobile ad hoc to support a stable operating environment for applications. [5] introduces the concept of EgoSpaces that are coordination models and middleware for mobile ad hoc networks to provide means for applications to adapt context changes occurring in dynamic environment. Their design goal is to provide a formal abstract approach to context-awareness and middleware managing an extended notion of context. [6] represents an architecture in which services are continuously evaluating system conditions in a self-organized manner to adjust service placement and capabilities.

The authors in [7] argue one of the main functions of future networks will be information delivery, and the underlying technology needs to disappear from the user's perspective. However future network will also be very diverse, and they will be managed by a large number of independent operators. Hence for transparency of the underlying technology control-plane interworking is required. [8] studies the reasons why IP-based QoS is not widely deployed, and concludes some main reasons for this is lack of integrated control and management, simplicity and measurable guarantees. [9] represents a P2P Wireless Network Confederation (P2PWNC) model, in which a set of administrative domains is providing wireless Internet access to each other's users. The authors aim to replace the human administrator of roaming agreements by Domain Agents (DA), thus eliminating administrative overhead.

While all of these research efforts address many critical issues, they do not fully address the emerging needs of future wireless and ubiquitous networks. They are problem statements, or they are focused on specific environment such as mobile 
ad-hoc networks. However, these coexisting different environments need to cooperate in the future, which is the main goal of our approach. We need to consider selforganized establishment of QoS, management of user and network mobility and other control functions in highly dynamic heterogeneous networks.

\section{Application Scenarios}

In this section, we discuss two applications scenarios, which illustrate the concept of network agreements in current and future networks. This will show why a new, generic and autonomous solution is needed for future ubiquitous networks.

Limitation of Current Roaming Agreements. Nowadays, a roaming agreement is established between two or more wireless operators outlining the terms and conditions under which the each operator will provide wireless service to the other's subscribers.

Roaming is usually associated with cellular mobile technologies, such as GSM (Global System for Mobile Communication), but it can also be applied to other type of wireless technologies, such as WLAN (Wireless Local Area Network). For instance, in [10], roaming between 802.11 networks and 3GPP networks is described. In its most simple form, the user of the 802.11 network is authenticated based on the SIM card in the 3GPP network. More advanced interworking, which is still to be defined, will also allow seamless handover between the two technologies, i.e. communications are interrupted when a handover is performed. However, with today's roaming agreements, services are not seamless for handover between operators, even if the handover is within the same technology.

The current concept of roaming agreements between operators is quite limited, since agreements are long-lived and commonly manually established for well-defined services between a pre-known set of commercial operators. Next generation networks however will enable a very large number of flexibly defined services in addition to those already known. These services will be offered by large operators as well as private users, in networks of distinct size from a PAN to a backbone network. There is a need to realize agreements concerning these services between networks. Users are "always on" and services can be accessed anywhere. Networks form dynamically, they move, and flexibly react to the users' needs. Such scenarios can only be handled if roaming agreement establishment becomes more dynamic, flexible and selforganized.

Network Agreements for Next Generation Networks. A future business man is using his PAN while traveling on a train that has its own network. The moving train network needs to establish connectivity with different access networks along the train track that can belong to different operators. The business man connects his entire PAN to the train network in a single step, and enrolls in a videoconference. He would like to go through the videoconference keeping the necessary quality level and without having to deal with on-the-fly configurations and agreements.

To allow the business man to move seamlessly, network functionality such as QoS, mobility, security and charging needs to be realized on-the-fly between train and 
access networks. Note these functionalities are not independent, as a handover may only be performed if adequate security credentials are provided, and deteriorating QoS may trigger handover etc. Such automatic realization of control-plane interaction between functionalities, and between heterogeneous, moving networks is not possible today, except in special-purpose, functionality- restricted cases.

\section{A Framework for Network Composition}

In this section, we introduce our new framework for network composition. The goal is to provide a flexible and extensible control plane, which can be composed in a selforganized way without manual intervention. We discuss the different kinds of network agreements and give a framework and architecture for managing the agreements. In the following, we first introduce the notion of network composition. Then we discuss different kinds of composition agreements and how to realize them. Also, the interfaces for network composition and architecture are presented. Here, we focus on the internal architecture to enable a flexible, efficient and extendible composition framework, which is not limited to specific services.

\subsection{Ambient Network, Ambient Control Space and Functional Areas}

An Ambient Network (AN) consists of one or more network nodes and/or devices. It has a common control plane called Ambient Control Space (ACS). Well-defined access to the ACS is provided to other ANs through the Ambient Network Interface (ANI). An AN has one or more identifiers, it can be contacted via the ANI, and it can compose with other ANs. The AN architecture is schematically shown in Figure 1.

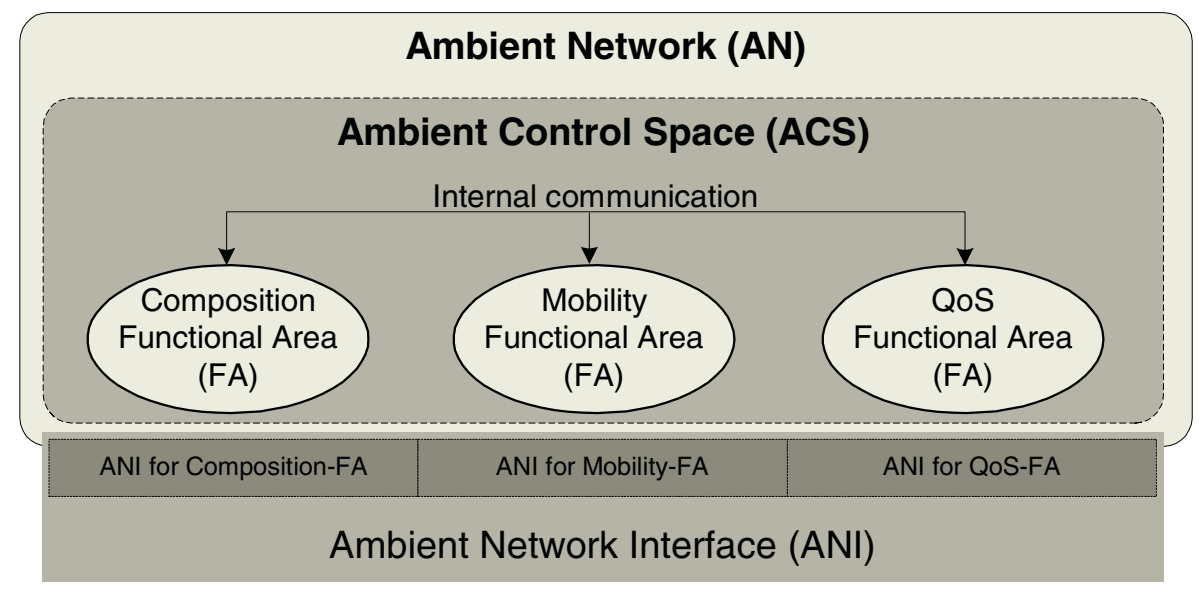

Fig. 1. An example of the Ambient Network Architecture

In the second example of Section 3, the business man's PAN is an AN comprising one or many devices with a joint control of the available resources. The train network 
is another AN and itself can be composed; e.g. each railway carriage is an $\mathrm{AN}$ and all of them are further composed to be a composed train AN with common control and certain edge nodes towards access networks along the track, see also Fig. 2. Each AN in this scenario has its own ACS. The ACS of the PAN is interacting with the ACS of the train AN via the ANI during a discovery and composition phases to gain access to train services and the Internet for all PAN devices.

There are minimal prescriptions how the ACS is realized, or what functionality it supports. It is organized into a number of so-called Functional Areas (FAs), which allow a grouping of topic-related control and management tasks. E.g. there could be a QoS-FA and a mobility-FA. A given FA integrates existing control functions and protocols, e.g. the mobility-FA includes mobile IP and Foreign Agents, however adds means for cooperation between FAs of the same and other ANs for realizing a composition. Particularly, all ANs have a Composition FA that orchestrates the input from all FAs from the same AN for a composition process.

In the second example presented in Section 3, the QoS-FAs of PAN and train network could agree that the train AN takes care of QoS control on behalf of the PAN. Correspondingly the mobility and security-FAs negotiate to transfer mobility and some security control so that the train network is able to do authentication to an access network on behalf of the PAN. As a result of roaming agreement between the train network and the access network, the train network may delegate some specific control functionalities to the PAN, e.g. it may instruct to perform specific priority packet marking for different traffic types to enable the necessary quality level.

\subsection{Ambient Network Interface and Generic Ambient Network Signaling}

The ANI is an open interface used by ANs to communicate with each other and therefore it is a network-network interface. Its main task is to enable efficient, and consistent message communications among FAs of the ACSs. This communication can take place during the composition negotiation, or inside a composed AN for communication between FAs. The ANI has to integrate existing legacy protocols and interfaces. When a new FA is added to an ACS, ANI will have to be extended to be able to support communication needs. To this end the ANI has a modular structure; each FA is implementing its own portion of the ANI as represented in Figure 1. The instantiation of the ANI may vary according to the ACS, for example a single logical ANI may be distributed over multiple physical network nodes each of them hosting a dedicated instance of a specific FA, or a single physical network node may implement the entire ANI. A distributed ANI implementation can be used to provide for example redundancy or load balancing.

The Generic Ambient Network Signaling (GANS) is the open base set of protocols enabling transport of signaling messages between FAs via the ANI. It is important to emphasize that GANS does not replace standard or de-facto standard protocols, which are used for instance to exchange routing information or for mobility support. GANS is used to exchange information currently not sufficiently covered by generally accepted protocols - e.g. SLA (Service Level Agreement) negotiation, capability exchange, and roaming agreement negotiation. In the example presented in Section 4, the QoS-FAs may start a negotiation using GANS to find out whether they support 
compatible protocols. When one such protocol has been found and agreed, they may to switch to use that protocol.
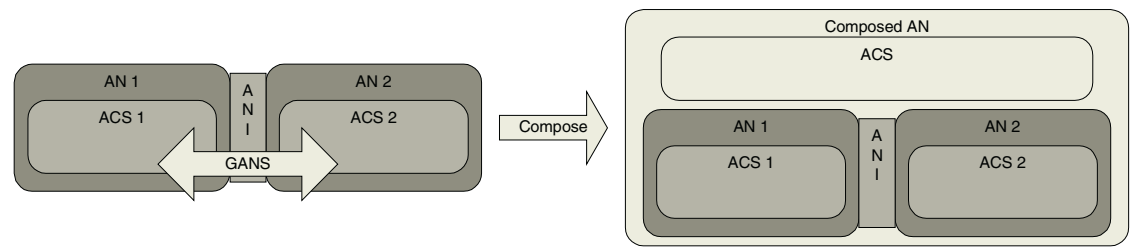

Fig. 2. An example of composition and resulted composed network

Figure 2 represents an example of how two ANs, AN 1 and AN 2, are composing using the GANS protocol to achieve control-plane interaction and correspondingly a composed AN with a joint ACS.

\subsection{The Concept of Self-organized Network Composition}

The concept of network compositions is introduced to support self-organized controlplane interworking of networks. It enables consistent management over cooperating networks and hides their interconnection details as well as internal structure to the outside. It improves network operation and service efficiency.

The details of control plane interworking between composing networks are fixed in a Composition Agreement. A composition establishment consists of the negotiation and then the realization of a Composition Agreement. Both negotiation of Composition Agreement and its realization should be autonomic i.e. they are usually triggered by internal processes and proceed with minimal user interaction.

Policies play an important role in the composition process. The decision whether to compose is policy-based, the negotiation of the Composition Agreement is policybased, and the Composition Agreement itself must meaningfully combine the policies of the composing ANs such that the composed AN has its own policies governing future compositions.

\subsubsection{Composition Agreements}

A Composition Agreements describes all mandatory and optional policies composing ANs agree to follow. A Composition Agreement is created when individual Ambient Networks agree with each other to compose. It is updated when all members of the composed network agree to change it. It exists as long as the composition exists, even when the members of that composition change.

A Composition Agreement is negotiated and created between all FAs of involved ANs. The structure of a Composition Agreement is modular with respect to the FAs. It consists of a general part specifying the basic rules all involved FAs have to follow and a number of subparts referring to agreements between individual FAs. Examples of the content of a Composition Agreement include: 
- $\quad$ Identifier of composed AN;

- IP address ranges;

- What resources in which networks are involved;

- Establishing and maintaining QoS of connectivity among individual networks;

- $\quad$ Security associations and trust relations among individual networks;

- Compensation/accounting;

- Common policies to outside and

- $\quad$ The way to realize the Composition Agreement (see more in the next section).

The Composition Agreement can describe a symmetric or an asymmetric sharing of resources, responsibilities, services, duties and permissions between networks involved. An example of a symmetric composition is several BANs composing to set up an ad hoc network, each of them playing a similar role in the composed network. An example of an asymmetric composition is a PAN in a train composing with the train's network to enjoy an entertainment program.

Composition Agreements are expected to often contain off-the-shelf components to improve performance. It is also possible to pre-establish Composition Agreements, or to re-use Composition Agreements negotiated earlier.

\subsubsection{Realization of the Composition Agreement}

A Composition Agreement can describe more or less tight cooperation of ANs. We loosely distinguish network integration, control sharing and network interworking.

With network integration, constituent ANs contribute all their logical and physical resources to the composed AN. They give up individual control of some resources and establish a joint ACS. They also hide their own identifiers such that they are not visible individually to the outside. In practice, this means that an AN can only be a member of one such composition at the time. The PAN in our example in Sec. 2 may be one example of network integration, when all its devices (e.g. laptop, PDA, mobile phone) have agreed to give up their individual identities and form a new composed network with a common control plane. Another example is the step-by-step integration and expansion of an infrastructure mobile communication network, where a group of equipment is typically installed and tested as a separate network and then integrated into the existing infrastructure network.

With control sharing, each constituent AN contributes only a part of its logical or physical resources to the composed network but keeps control over the rest. Control of these resources may be delegated to FAs of particular constituent AN, or a joint ACS may be established. An individual network may participate in multiple such compositions in parallel. An example of control sharing are several PANs that build a dynamic ad-hoc network for a meeting, or the delegation of authentication and authorization of the PAN to a train network as represented in our example in Sec. 3.2.

With network interworking, the individual FAs of each constituent AN just coordinate their work. E.g. in roaming agreements, they agree users are always authenticated in the home network. 


\subsubsection{Composition Functional Area}

The Composition Functional Area (C-FA) is an addition to existing control-plane functionality. Its role is the coordination of the FAs of a single AN. It also contains decision logic for running and controlling the composition process. For example, the C-FA collects triggers from other FAs that a composition should be attempted, and takes care all FAs participate in the negotiation and realization of the Composition Agreement. There are minimal assumptions about the ways C-FA may operate, be implemented or managed except its existence. A "Master C-FA" that actively drives the composition process based on policies may be rather straight-forward to implement. Another extreme is a "passive C-FA" that just collects input from other FAs, posts it for others to read, and makes sure it is consistent. However even a passive CA needs to have a logic that drives it to react on certain input. E.g. when it receives a trigger that composition should be attempted, it should make sure a decision is reached on the Composition Agreement in a timely fashion.

\subsubsection{Composition Creation}

A composition with an active "Master C-FA" could schematically proceed as follows: AN X discovers AN Y, e.g. by receiving a radio beacon, or by user interaction, and learns the identity of AN Y. Upon learning about the discovery, the C-FA of AN X consults a policy data base and finds out composition with AN Y should be attempted. Alternatively, e.g. the mobility-FA could prompt composition by reporting to the CFA deteriorating quality of the current path. Connectivity for control-plane signaling is established, and usually the security-FA authenticates AN Y by interacting with its peer security-FA in AN Y. Now the C-FA finds out what Composition Agreement it could offer. There may be a pre-established or off-the-shelve Composition Agreement attached to the identifier of AN Y. Otherwise, all FAs need to contribute to the creation of the agreement. The Composition Agreement is offered and negotiated with AN Y. Once the agreement is settled, the security-FA needs to authorize AN Y. Finally, the Composition Agreement is realized.

Three other procedures are needed to realize compositions, namely Composition Extension, which is used by individual ANs to join an existing Composed Network; Composition Agreement Update, which is used by members of a Composed Network to update the Composition Agreement; and Decomposition, which is used by an AN to leave a composition.

\section{QoS and Mobility Composition for Self-organized Roaming}

This section describes in more detail the usage of the composition framework. In the next generation networks scenario described in Section 3, the business man needs to obtain Internet access for all devices of his PAN in a single step. Moreover, for the video conference he also needs end-to-end QoS, which should be maintained while the train moves, by connecting to different infrastructure access networks along the train track and in stations. The entire process should proceed self-organized with minimal user interaction. Figure 3 illustrates the compositions relevant in this scenario. 


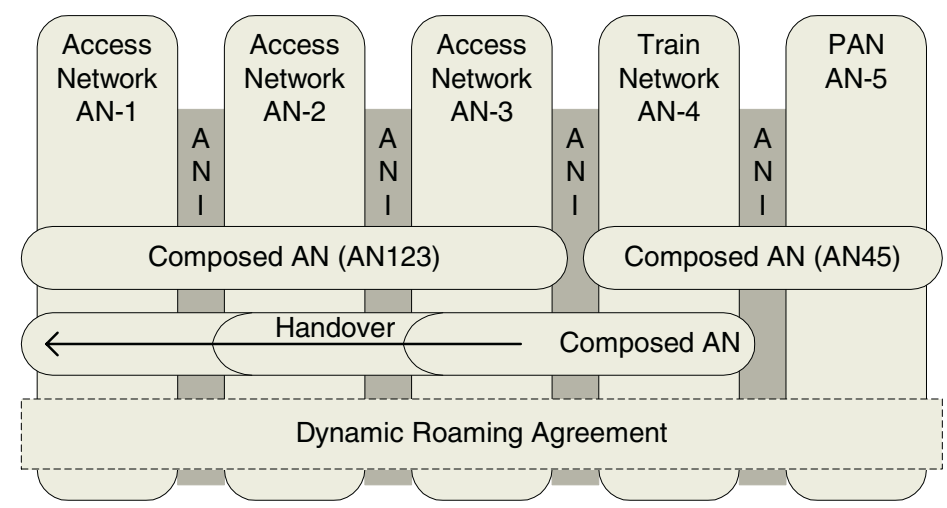

Fig. 3. Compositions in the "business man on a train scenario"

The PAN (AN-5) composes with the train network (AN-4). The composition is of the "control-sharing type". The Composition Agreement states that the train network will provide Internet access to all the devices included in the PAN, independent of its location inside the train, and the train network performs QoS and mobility control as well as some security control on behalf on the PAN, allowing it to be connected without having any perception of movement of the train and its own movement inside the train. By delegating some QoS control, the PAN authorizes the train network to negotiate QoS with the different access networks along the track, and to adapt multimedia sessions to network quality oscillations on its behalf. For mobility control, the train network may e.g. provide a translation service between the care-of addresses of the PAN seen outside the train, and a fixed address that is used by the PAN inside the train. By delegating mobility control, the PAN also authorizes the train network to authenticate it with each access network.

Because the devices of the PAN are composed to an integrated network, the train network only needs to negotiate with one entity, the PAN, rather than with all its constituent devices. While this control plane abstraction is more flexible, it also reduces the signaling load within the train network.

Along the train track, access networks (AN-1, AN-2 and $\mathrm{AN}-3$ ) compose to provide seamless communication services to the users of the train network, by creating a virtual access network. This composition is of the control-sharing type, in which access networks share logical and physical resources in order to jointly manage QoS and mobility within the virtual access network. The virtual access network delegates access control to the train network, by trusting all users the train network trusts. Joint mobility management may look as follows:

- Access networks may agree to implement a type of inter-network Fast Mobile IP [11], by allowing each network to have pre-configured address information, which reduces control signaling during handover, and eliminates address resolution time. 
- They may agree to implement a common Hierarchical Mobile IP [12] scheme, in which a hierarchic of mobility anchor points is jointly used in all access networks.

- Access networks can exchange information about their network capacity and number of clients, or even may combine their context handling schemes in order to allow a wiser decision about the next attachment point.

Regarding QoS, the access networks may agree to establish a consistent QoS management for the composed network. For instance, class-based networks may agree to use the same type of classes, and to exchange information about the usage of resources within each class. This will, for instance, allow admission control to be done only at the edges of the virtual access network and reduce the time required to set up requested QoS levels, contributing to the seamless movement of multimedia sessions.

Since the train may move fast, a network inter-working type composition may be established between the train and the virtual access network. The Composition Agreement describes how QoS and mobility control is handled between the train network and the virtual access network, without having any sharing of control between them. The Composition Agreement presumably is pre-established, since trains of this company frequently travel on these tracks.

In terms of interaction of FAs this composition process could e.g. proceed as follows: Composition may e.g. be triggered by the mobility-FA detecting the virtual access network is in reach. A policy tells it composition should be attempted. This information is relayed to the C-FA, which draws off-the-shelve the well-known Composition Agreement and informs all other FAs composition should be performed according to this agreement.

\section{Conclusions}

We have explained the new framework of Ambient Networks and composition, which aims to support the ubiquitous, heterogeneous mobile networking vision. We argue that our new abstractions, nodes as networks and network composition, give a more coherent and simplified view for future control architectures. We illustrated the need for self-organized dynamically configurable control planes, particularly for network interworking. The concept of composition aims to provide such interworking.

The composition concept proposed in this paper can include e.g. the TurfNet architecture [13]. TurfNet describes an inter-domain communication mechanism that does not require global network addressing or a common network protocol. Hence, it provides an approach to solve the problem of composition of address spaces and inter-domain routing. The different types of network composition considered by TurfNet, namely horizontal and vertical composition, map to our terms network integration and control sharing / network interworking respectively. 
The Ambient Networks approach is essential for ubiquitous environments for several reasons.

- The responsibility for network control functions such as QoS and mobility should not be placed on the end system (edge) alone, especially for limited, wireless devices, possibly without user interfaces, in a highly dynamic environment. With composition, control functions can be explicitly assigned and distributed.

- Mobile networks will need a much larger variety of control plane interworking options than possible with static network agreements and fixed protocol solutions.

- Dynamic internetworking is simplified if the procedure is independent of the nature of the entities involved. It shouldn't matter whether a single device, a PAN or the mobile network of a train (itself containing terminals and PANs) is attached to an access network: An Ambient Network can be a single node, a network, or a network of networks. Composition always proceeds according to the same procedure.

- The configuration of control-plane interaction needs to become an autonomic process, because it is very complex and yet needs to be realized on-the-fly, and moreover transparently to the user.

We have shown a control plane framework, which is extendible based on the concepts of functional areas. Furthermore, we have presented design guidelines for a generic signalling protocol for network composition, which coordinates the individual negations of the FAs. The protocol development is still in very early stage. For the composition, we envisage different degrees ranging from loose interworking over control sharing to network integration.

Several issues have not been detailed in this paper for lack of space, e.g. addressing and discovery of ANs. Our current work within the Ambient Networks project aims to detail and validate the framework presented here.

Acknowledgements. This work is supported by the Ambient Networks Project, partially funded by the European Commission under its Sixth Framework Programme. It is provided "as is" and without any expressed or implied warranties, including, without limitation, the implied warranties of fitness for a particular purpose. The views and conclusions contained herein are those of the authors and should not be interpreted as necessarily representing the official policies or endorsements, either expressed or implied, of the Ambient Networks Project or the European Commission.

\section{References}

[1] http://www.ambient-networks.org

[2] N. Niebert, A. Schieder, H. Abramowicz, G. Malmgren, J. Sachs, U. Horn, Ch. Prehofer and H. Karl, ,Ambient Networks: An Architecture for Communication Networks beyond 3G“, IEEE Wireless Communications, April 2004.

[3] D. Clark, C. Partridge, Ch.s Ramming, and J. Wroclawski, "A Knowledge Plane for the Internet", ACM Sigcomm 2003, Karlsruhe, Germany, Aug. 2003. 
[4] K. Herrmann, K. Geihs, and G. Mühl, "A Self-Organizing Infrastructure for Mobile Commerce" KiVS 2003.

[5] C. Julien, G.-C. Roman, "Egocentric Context-Aware Programming in Ad Hoc Mobile Environments", Proc. 10th ACM SIGSOFT Symposium on Foundations of Software Engineering, Charleston, SC, USA, 2002.

[6] S. Graupner, A. Andrzejak, V. Kotov, H. Trinks, “Adaptive Control Overlay for Service Management", 1st Workshop on the Design of Self-Managing Systems (AASMS'03), San Francisco, 2003.

[7] R. O'Connor, S. van der Meer, "Present and future organisational models for wireless networks", Proc. 1st Intl. Symposium on Information and Communication Technologies, Dublin, Ireland, 2003.

[8] L. Burgstahler, K. Dolzer, C. Hauser, J. Jähnert, S. Junghans, C. Macián, W. Payer: “The Missing Pieces for QoS Success: What have we learned, why do we care?" ACM SIGCOMM workshop on Revisiting IP QoS, Karlsruhe, Germany, 2003.

[9] Elias C. Efstathiou, George C. Polyzos: "A peer-to-peer approach to wireless LAN roaming", Proc. 1st ACM Int. workshop on Wireless mobile applications and services on WLAN hotspots, San Diego, CA, 2003.

[10] 3GPP TS 23.234 "3GPP system to Wireless Local Area Network (WLAN) interworking; System description”, v2.4.0 Jan. 2004.

[11] R. Koodli, "Fast Handovers for Mobile IPv6", IETF, Internet-Draft, March 2003, work in process.

[12] H. Soliman, et al, "Hierarchical Mobile IPv6 Mobility Management", IETF, InternetDraft, June 2003, work in progress.

[13] S. Schmid, L. Eggert, M. Brunner, J. Quittek, „TurfNet: An Architecture for Dynamically Composable Networks“ Proc. 1st IFIP Int. Workshop on Autonomic Comunication, Berlin, Oct. 2004. 